\title{
Associação entre domínio de capacidade funcional (SF-36), medo de cair e histórico de quedas em idosos ativos
}

\author{
Fernanda Pinheiro Cardoso ${ }^{1}$ \\ Andréa Kruger Gonçalves²
}

\section{RESUMO}

Objetivos: Este estudo teve como objetivo verificar a associação entre domínio de capacidade funcional, medo de cair, idade e histórico de quedas de idosos ativos. Metodologia: Estudo descritivo, quantitativo. A amostra $(n=152)$ foi dividida em idosos caidores $(n=28)$ e idosos não caidores $(n=124)$, onde se utilizou do domínio de capacidade funcional do questionário de Avaliação de Qualidade de Vida (Brasil SF-36), da escala de eficácia em quedas - Internacional - Brasil (FES - I - Brasil) e do relato de ocorrência de quedas. Para a relação dos dados se utilizou da Correlação de Pearson no programa SPSS 17.0. Resultados: Pode-se observar que embora tenha sido apresentada significância estatística, a correlação de Pearson não mostrou relação significante entre o domínio de capacidade funcional, o medo de cair e a idade na comparação dos grupos de caidores e não caidores. Dessa maneira a hipótese de que a prevalência de quedas poderia influenciar na capacidade funcional e no medo de cair não pode ser confirmada. Considerações finais: A amostra estudada identificou baixa prevalência de quedas, os grupos apresentaram um bom nível de independência funcional. Os valores médios obtidos através dos questionários são muito semelhantes, ligeiramente mais favoráveis aos idosos não caidores, no entanto, ambos apresentaram preocupação com a queda.

PALAVRAS-CHAVE:

Envelhecimento. Capacidade Funcional. Quedas.

\footnotetext{
1 UFRGS
}

2 UFRGS. 


\section{ABSTRACT}

Objectives: This study aimed to determine the association between functional capacity domain (SF-36), fear of falling, age and history of falls of active seniors. Methodology: descriptive, quantitative study. The sample $(n=152)$ was divided into fallers $(n=28)$ and elderly non-fallers $(n=124)$, in which we used the functional capacity domain of the Quality of Life Assessment questionnaire (SF-36 Brazil) the effectiveness of scale falls - International - Brazil (FES - I - Brazil) and the reporting of falls. For a list of the data was used the Pearson correlation in SPSS 17.0. Results: It can be observed that although it was presented statistical significance, the Pearson correlation showed no significant relationship between functional capacity domain, the fear of falling and the age when comparing fallers and non-fallers groups. Thus the hypothesis that the prevalence of falls could influence the functional ability and fear of falling can not be confirmed. Final Thoughts: The study identified low prevalence of falls, the groups presented a good level of functional independence. The mean values obtained from the questionnaires are very similar, slightly more favorable to non-fallers, however, both showed concern about the fall.

\section{KEYWORDS:}

Aging; Functional Capacity; Falls. 


\section{Introdução}

O envelhecimento de uma população é resultante de progressos no sistema de saúde, refletindo em melhorias nas condições de vida dos habitantes e repercutindo em uma das maiores conquistas culturais de um povo em seu processo de humanização. Segundo a Secretaria dos Direitos Humanos (2012), em 2050, os idosos atingirão $22 \%$ da população mundial, sendo projetado o número de 2 bilhões de pessoas. Conforme dados do IBGE, em 2012, a população idosa atingia $11,5 \%$ do número de habitantes no mundo. No entanto, o alargamento do número de idosos leva a um grande problema de saúde pública, pois, em decorrência dos efeitos deletérios inerentes do processo de envelhecimento humano, tem-se como agravante à saúde destes a suscetibilidade a eventos de queda (CARVALHO et al., 2013).

A queda é considerada como a perda da estabilidade do corpo, decorrente de uma série de fatores. Estimada como não intencional, ela ocorre quando o indivíduo é incapaz de recuperar o equilíbrio e cai, ao nível do chão ou mais baixo, podendo estar relacionada à insuficiência súbita ou mesmo de mecanismos neurais e causando prejuízos à saúde dos idosos (RODRIGUES et al., 2013). As quedas podem ter como fatores causais diversos elementos, bem como interações medicamentosas, a não utilização de órteses prescritas, declínios de força e massa corporal magra, perda de condicionamento físico, dentre outros. Segundo o Ministério da Saúde (2012), 30\% dos idosos caem ao menos uma vez por ano, e, para aqueles acima de 80 anos, a ocorrência de quedas aumenta para 50\%. Devido aos declínios decorrentes dos eventos de quedas, estima-se que $13 \%$ destes são "caidores" recorrentes.

A mais severa das consequências decorrentes das quedas é a fratura, em que o idoso fica restrito ao leito, propiciando o desenvolvimento de problemas respiratórios, vasculares e, nos casos de internação, favorecendo a infecção hospitalar, o que aumenta o risco de óbito, já que, em média, $30 \%$ dos idosos que fraturam o fêmur morrem em um ano (CARVALHO et al., 2013).

Os mesmos autores apontam ainda que esses eventos contribuem para o aumento dos custos com cuidados médicos, uma vez que estão associados a uma série de limitações físicas e psicológicas. Conforme dados do Ministério da Saúde (2012), o Sistema Único de Saúde (SUS) registra, a cada ano, o gasto de 51 milhões de reais com o tratamento de fraturas decorrentes de queda.

Quanto às limitações psicológicas, o medo de cair tem sido reconhecido como um importante fator que está associado à preocupação com um possível evento de queda, influenciando na qualidade de vida e podendo refletir negativamente na capacidade funcional, uma vez que estes indivíduos, por medo de cair, tornam-se menos ativos e gradativamente mais sedentários. No entanto, fatores psicológicos, bem como ansiedade e depressão, aumentam a predisposição de idosos a desenvolverem a ptofobia, uma reação fóbica relacionada ao medo de cair, em que o indivíduo não consegue manter-se em pé e caminhar sem apoio, mesmo não havendo nenhuma anormalidade neurológica ou osteoarticular (GAl et al., 2009).

Patil et al. (2013) apontam que não raro o medo de cair acaba por desencadear um evento de queda, ocasionando piora na capacidade funcional. Entende-se por capacidade funcional a habilidade de o indivíduo desempenhar atividades básicas, bem como banhar-se e vestir-se, e atividades instrumentais da vida diária, que são compreendidas como habilidade de viver independentemente dentro da comunidade, gerindo de maneira independente sua casa, suas finanças e suas medicações (DIAS et al., 2011).

Com o avançar da idade, devido aos processos deletérios decorrentes do envelhecimento humano, é comum identificar parâmetros reduzidos de força e massa óssea, tornando-os mais frágeis e suscetíveis a quedas, devido a má postura, fragilidade muscular e equilíbrio prejudicado. As perdas de força e de equilíbrio, de maneira geral, acarretam em prejuízos à capacidade funcional de idosos e, quando somadas ao histórico de doenças ao longo da vida, inatividade física e quedas, ocasionam aumento na dependência para realizar suas atividades de vida diária (GASPAROTTO et al., 2014).

Existe uma relação mútua entre a capacidade funcional, o medo de cair e as quedas, em que ambos impactam diretamente na qualidade de vida dos en- 
velhescentes. Estudar os fatores associados ao medo de cair, assim como a capacidade funcional e quedas, é uma via de pesquisa importante para o entendimento da perda da independência e eventos de quedas, de forma que se possa identificar o perfil de idosos caidores, beneficiando-os com intervenções preventivas.

Portanto, o presente artigo tem como objetivo analisar a associação entre o medo de cair com o domínio de capacidade funcional e a prevalência de quedas de idosos ativos pertencentes a um grupo de programa de exercícios físicos da Escola Superior de Educação Física (ESEF) da Universidade Federal do Rio Grande do Sul (UFRGS).

\section{Metodologia}

A amostra da pesquisa foi composta por 152 idosos (com idade entre 60 e 90 anos) integrantes do Centro de Esportes, Lazer e Recreação do Idoso (CELARI), sediado na ESEF da UFRGS. Os dados são oriundos do banco de dados do centro e foram cedidos pela coordenação. Foram utilizados os dados daqueles que possuíam todos os instrumentos necessários para o estudo. A coleta dos dados ocorreu em março de 2014.

O referido programa foi criado no ano de 1999 e oferece a prática de atividades físico-educativas, envolvendo profissionais de diversas áreas da saúde. São oferecidas diferentes modalidades de atividades físicas e de cunho socioeducativo, compreendendo atividades aquáticas, ginástica, dança, musculação, grupo de percussão e de canto, além de oficinas de equilíbrio, cultural, literária e de jogos lógicos. O programa atende pessoas com idade superior a 55 anos, de ambos os sexos, sendo que, na ocasião da coleta de dados, havia 210 pessoas matriculadas.

Para analisar a capacidade funcional dos idosos, foi utilizado o escore do domínio de capacidade funcional do Questionário de Avaliação de Qualidade de Vida (Brasil SF-36), Ciconelliet al. (1999). Esse instrumento é composto por uma escala multidimensional com 36 itens referentes à saúde divididos em 8 domínios: capacidade funcional, aspectos físicos, dor, estado geral de saúde, vitalidade, aspectos sociais, aspectos emocionais e saúde mental. Cada domínio é calculado a partir do rawscale, o qual considera o valor mínimo e o máximo de cada domínio, além da sua variação. $O$ resultado pode variar entre 0 e 100, sendo que, quanto maior o valor, melhor é a condição de saúde. Para este estudo, utilizou-se o Domínio Capacidade Funcional (CF).

O CF é composto por 10 questões referentes às atividades de vida diária que avaliam o nível de dificuldade de execução em atividades que variam desde vigorosas, que exigem muito esforço para realização, atividades moderadas, bem como limpeza da casa, levantar e carregar mantimentos, subir lances de escada, curvar-se, caminhadas longas e curtas, a até mesmo tomar banho sozinho. $\mathrm{O}$ avaliado devia responder as questões do CF através da seguinte situação: "Devido a sua saúde, você tem dificuldades para fazer essas atividades?". Essa questão foi respondida, gerando a seguinte pontuação: 1 para as que são muito difíceis; 2 para as que são um pouco difíceis; e 3 para as que o avaliado não encontra dificuldade para realizá-las. A pontuação máxima de rawscale para este domínio é de 85 pontos, situação em que o avaliado é considerado funcionalmente independente, uma vez que não são relatadas dificuldades para realizar atividades físicas vigorosas.

Para aferir o medo de cair, utilizou-se a Escala de Eficácia de Quedas - Internacional - Brasil (FES-I-Brasil) Camargos et al. (2010), que apresenta 16 situações que avaliam a preocupação com o medo de cair. Os pesquisados deveriam avaliar as situações como se estivessem realizando-as e apontar como se sentiria em relação a quedas. As respostas variaram de "nem um pouco preocupado", "um pouco preocupado", "muito preocupado" e "extremamente preocupado", gerando um escore de 1 a 4, respectivamente. $\mathrm{O}$ escore total obtido com o questionário tem como menor valor 16 , que corresponde a nenhuma preocupação, e valor máximo 64, em que o avaliado apresenta extrema preocupação com quedas para as atividades apresentadas na escala.

Delbaere et al. (2010) apontaram em seu artigo um ponto de corte para os escores obtidos na FES, no qual é considerada "baixa preocupação com a ocorrência de quedas" a pontuação menor ou igual 22 ( $\leq 22)$ e "alta preocupação com a ocorrência de quedas" a pontuação maior que 22 (>22). 
Para o estudo, também foram coletadas informações referentes à ocorrência de quedas nos últimos 6 meses, bem como à quantidade e à ocorrência de fratura.

Os instrumentos de pesquisa eram autoaplicáveis, tendo sido supervisionados pelos bolsistas do projeto. Este artigo foi elaborado a partir do banco de dados do CELARI. Todos os participantes assinaram o Termo de Consentimento Livre e Esclarecido antes da coleta dos dados. E o estudo desenvolvido pelo CELARI foi aprovado pelo Comitê de Ética em Pesquisa do Instituto de Psicologia, sob protocolo número 2010036.

Este é um estudo descritivo quantitativo, no qual a análise estatística foi realizada através do Teste de Correlação de Pearson, no programa SPSS 17.0, com nível de significância de $p \leq 0,05$.

Os idosos foram divididos em dois grupos, os que já sofreram queda (grupo $A$ ) e os que não sofreram queda (grupo B). Foram correlacionadas as pontuações de cada grupo do CF do questionário (SF-36) com a FES-I. Também foram associados a idade dos integrantes com CF e o FES-I com a idade.

\section{Resultados}

O grupo de participantes do estudo foi constituído por 132 mulheres e 20 homens, sendo que, destes, 28 indivíduos já tiveram pelo menos um evento de queda e 124 não apresentaram evento de queda até a data da coleta dos dados. A idade média da amostra foi de $71,3( \pm 6,9)$ anos, sendo o mais novo com 60 anos, e o mais velho com 90 anos.

No CF, o grupo A atingiu escore médio de 67,6 $( \pm 22,6)$, e o grupo $B, 71,8( \pm 20,9)$. Já na FES-I, o grupo A obteve como escore médio $26,8( \pm 9)$, e o grupo $B, 22,5( \pm 4,9)$.

Os dados podem ser visualizados na tabela a seguir (Tabela 1).

Tabela 1 - Comparação de idade (anos), CF e FES entre os grupos

\begin{tabular}{|c|c|c|c|c|c|c|}
\hline \multicolumn{3}{|c|}{ IDADE } & \multicolumn{2}{|l|}{$\mathrm{CF}$} & \multicolumn{2}{|l|}{ FES } \\
\hline & MÉDIA & DP & MÉDIA & DP & MÉDIA & DP \\
\hline GRUPO A & 73,6 & $\pm 8,3$ & 67,6 & $\pm 22,6$ & 26,8 & \pm 9 \\
\hline GRUPO B & 70,9 & $\pm 6,5$ & 71,8 & $\pm 20,9$ & 22,5 & $\pm 4,9$ \\
\hline
\end{tabular}

CF- Domínio de Capacidade Funcional; FES- Escala de Eficácia em Quedas; DP- Desvio Padrão.

Quanto à associação do escore do CF com o FES- I, o grupo A obteve $r=-0,28$, o que indica associação significativa estatisticamente de correlação negativa baixa, e o grupo B $r=-0,43$, o que indica associação significativa estatisticamente de correlação negativa moderada. Na correlação do escore do CF com a idade, o grupo A apresentou valor $r=0,11$, relação significativa estatisticamente, com valor de correlação positivo baixo, e o grupo $B r=-0,21$, estatisticamente significativo de correlação negativa baixa. Ao relacionar o FES-I com a idade, o grupo A apresentou valor $r=0,04$, e o grupo $B r=0,09$, ambas relações apresentando correlação positiva baixa, porém com significância estatística.

Na Tabela 2, são apresentados os valores decorrentes da correlação dos dados, conforme segue:
Tabela 2 - Resultados obtidos na correlação dos dados

\begin{tabular}{l|l|l}
\hline & GRUPO A & \multicolumn{1}{l}{ GRUPO B } \\
\hline CF X FES-I & $-0,28$ & $\mathrm{R}$ \\
\hline CF X IDADE & 0,11 & $-0,43$ \\
\hline FES-I X IDADE & 0,04 & $-0,21$ \\
\hline
\end{tabular}

CF- Domínio de Capacidade Funcional; FES- Escala de Eficácia em Quedas;

$\mathrm{r}=$ Relação.

\section{Discussão}

Este estudo identificou que, em um grupo de 152 idosos ativos, apenas 28 indivíduos (18,4\%) relataram algum evento de queda, indicando baixa prevalência de caidores. Estudos que investigam idosos ativos relatam percentuais mais baixos quando se 
referem às quedas (BECK et al., 2011). Padoin et al. (2010) corroboram com os achados, relatando que, em uma amostra de idosos fisicamente ativos, o risco de ocorrerem quedas é reduzido através da manutenção da capacidade funcional.

Quanto aos escores de $\mathrm{CF}, 85$ pontos é o valor máximo que pode ser atingido, indicando que o indivíduo realiza de maneira independente atividades básicas e instrumentais da vida diária. Os valores médios obtidos para ambos os grupos foram elevados, o grupo A atingiu como média $79,5 \%$ $(67,6 \pm 22,6)$ da pontuação máxima, e o grupo B $84,5 \%(71,8 \pm 20,9)$. Os achados apontam que mesmo o grupo com histórico de quedas apresenta bom nível de CF, provavelmente por se tratar de uma amostra fisicamente ativa, no entanto, o valor médio de CF é ligeiramente menor para o grupo que sofreu queda. Brito et al. (2013) confirmam isso ao relatarem que, após um evento de queda, os idosos de modo geral têm perdas de condicionamento, acarretando em prejuízos à capacidade funcional.

O medo de cair normalmente surge após a ocorrência de uma queda, e, neste estudo, pôde ser constatado que ambos os grupos manifestaram preocupação com a ocorrência. $O$ valor médio atingido no FES é ligeiramente maior para o grupo $A$ $(26,8 \pm 9)$ do que para o grupo $B(22,5 \pm 4,9)$.

$\mathrm{Na}$ associação do $\mathrm{CF}$ com o FES, ambos os grupos apresentaram correlação inversamente proporcional, ou seja, quanto maior é o $C F$, menor é a preocupação com a queda. No entanto, o grupo B apresentou valor de correlação mais significativo, tornando essa hipótese ainda mais forte. Declínios em CF e a preocupação com a queda são considerados como risco de queda no estudo de Patil et al. (2013) realizado com finlandesas idosas e fisicamente ativas, o qual apontou que, quanto pior a classificação de CF, maior era a preocupação com queda.

Quanto à associação de CF com a idade, na comparação entre os grupos, ambos apresentaram baixa correlação, porém o grupo B apresentou correlação negativa baixa, e o grupo $A$ positiva baixa. Para a associação do grupo $B$, à medida que o indivíduo envelhece, ocorre piora na capacidade funcional, uma vez que são inversamente proporcionais. Já na correlação do grupo A, o escore do CF aumenta proporcionalmente com a idade. Nem todos os eventos de queda são limitantes, possivelmente os eventos de quedas sofridos pela amostra não tenham acarretado em limitações, provavelmente pelo fato de que os envolvidos no estudo foram beneficiados através de intervenções preventivas antes da deteriorização funcional.

A capacidade funcional tende a diminuir com o passar dos anos, no entanto, a prática de exercícios físicos influencia positivamente nos níveis de independência (SILVA et al., 2010). Nogueira et al. (2010) referiram em seu estudo que $71 \%$ dos idosos apresentaram uma boa capacidade funcional, e, no de Ferreira et al. (2012), todos os idosos envolvidos na pesquisa se mostraram independentes para as atividades básicas e citaram que, quanto mais ativo é o indivíduo, menores são as limitações acarretadas ao longo do processo de envelhecimento.

$\mathrm{Na}$ associação do FES com a idade, a correlação resultou em positiva baixa para ambos os grupos, indicando que, quanto mais velho é o indivíduo, maior é a prevalência do medo de cair, mesmo para aqueles que não sofreram quedas. Beck et al. (2011) relatam que a ocorrência de quedas tende a aumentar linearmente com o aumento da idade. Conforme Gai et al. (2009), esse aumento ocorre mesmo que o idoso não tenha caído.

Mesmo que o idoso tenha um bom condicionamento físico, o medo de cair pode ser também associado a outros processos deletérios irreversíveis do envelhecimento humano, bem como a modificações estruturais e funcionais no sistema sensorial, que, mediante a sua gravidade, podem acarretar em limitações importantes, influenciando na perda de equilíbrio e em uma eventual queda.

Pode-se observar que, embora tenha sido apresentada significância estatística, a correlação de Pearson não mostrou relação significante entre $\mathrm{O} C$, o FES e a idade na comparação dos grupos A e B. Dessa maneira, a hipótese de que a prevalência de quedas poderia influenciar na capacidade funcional e no medo de cair não pôde ser confirmada.

A amostra estudada identificou baixa prevalência de quedas e que ambos os grupos, A e B, são fisicamente ativos e dispõem de um bom nível de independência funcional. Os valores médios obtidos através dos questionários são muito semelhantes, embora ligeiramente mais favoráveis aos idosos não caidores. Tanto o grupo A quanto o grupo B apresentaram preocupação com a queda, indicando que, mesmo que o 
idoso não tenha caído,ele acaba desenvolvendo a preocupação.Uma explicação para isso é que pode ser um mecanismo de proteção.

\section{Considerações Finais}

O presente estudo identificou baixa prevalência de quedas em uma amostra de idosos ativos, no entanto, ficou evidente que mesmo os idosos não caidores têm preocupação com possíveis eventos de queda. Os indivíduos da amostra apresentaram valores semelhantes para todas as variáveis. E, embora os idosos sem queda tenham obtido valores de média para idade, escore do CF e medo de cair um pouco mais favoráveis, o teste de Correlação de Pearson não indicou relação significativa entre a capacidade funcional e a presença de quedas, embora a estatística tenha apresentado significância. 


\section{Referências}

BECK, Amanda Pacheco et al. Fatores associados às quedas entre idosos praticantes de atividades físicas. Revista Texto Contexto de Enfermagem, Florianópolis, v. 20, n.2, p. 280286, abril/junho, 2011.

BRASIL. Secretaria de Direitos Humanos (Org.). Pessoa Idosa: Dados estatísticos. 2012. Disponível em: <http://www. sdh.gov.br/assuntos/pessoa-idosa/dados-estatisticos $>$. Acesso em: 19 fev. 2015.

BRASIL. Portal Brasil (Org.). Saúde: Quedas. 2012. Disponível em: <http://www.brasil.gov.br/saude/2012/04/quedas >. Acesso em: 19 fev. 2015.

BRITO, Thaís Alves et al. Quedas e capacidade funcional em idosos longevos residentes em comunidade. Revista Texto Contexto de enfermagem, Florianópolis, v. 22, v. 1, p. 43-51.

CAMARGOS, Flávia Fernandes Oliveira et al. Adaptação transcultural e avaliação das propriedades psicométricas da Falls Efficacy Scale - Internacional em idosos brasileiros (FES -I-BRASIL). Revista Brasileira de Fisioterapia, São Carlos, v. 14, n. 3, p. 237-243, maio/junho, 2010.

CARVALHO, Eliana et al. Relação entre as quedas, o equilíbrio funcional e a qualidade de vida em idosos. Revista Ensaios e Ciência: Ciências Biológicas, Agrárias e da Saúde, Campo Grande, v. 17, n. 1, p. 43-52, 2013.

CICONELLI, Rozana Mesquita et al. Tradução para a língua portuguesa e validação do questionário genérico de avaliação de qualidade de vida SF-36 (Brasil SF-36). Revista Brasileira de Reumatologia, Brasil, v. 39, n.03, p. 143-150, 1999.

DIAS, Rosângela et al. Características associadas à restrição de atividades por medo de cair em idosos comunitários. Revista Brasileira de Fisioterapia, São Carlos, v. 15, n. 11, p. 406-413, 2011
DELBAERE, Kim et al. The falls efficacy scale international (FES-I). A comprehensive longitudinal validation study. Age and Aging, v. 39, p. 210-216, 2010.

FERREIRA, Olívia Galvão et al. Envelhecimento ativo e sua relação com a independência funcional. Revista Texto Contexto de enfermagem, Florianópolis, v. 21, n. 3, p. 513518, 2012.

GAI, Juliana; GOMES, Lucy; CÁRDENAS, Carmen Jansen de. Ptofobia: o medo de cair em pessoas idosas. Revista Acta Médica Portuguesa, Portugal, v. 22, n. 1, p. 83-88, 2009.

GASPAROTTO, Lívia Pimenta Renó; FALSARELLA, Gláucia Regina; COIMBRA, Arlete Maria Valente. As quedas no cenário da velhice: conceitos básicos e atualidades da pesquisa. $R e-$ vista Brasileira de Geriatria e Gerontologia, Rio de Janeiro, v. 17, n. 1, p. 201-209, 2014.

NOGUEIRA, Silvana et al. Fatores determinantes da capacidade funcional em idoso longevos. Revista Brasileira de Fisioterapia, São Carlos, v. 14, n. 4, p. 322-329, jul./ago., 2010.

PADOIN, Priscila Gularte et al. Análise comparativa entre idosos praticantes de exercício físico e sedentário quanto ao risco de quedas. O mundo da Saúde, São Paulo, v. 34, n. 2, p. 158-164, 2010.

PATIL, Radhika et al. Concern about falling in older women with a history of falls: associations with health, functional ability, physical activity and quality of life. Gerontology, Finlândia, v. 60, p. 22-30, 2014.

RODRIGUES, lara Guimarães; LIMA, Margareth Guimarães; BARROS, Marilisa Berti de Azevedo. Falls and health-Related quality of life (SF-36) in ederly people - ISACAMP 2008. Health, v. 5, n. 12a, dezembro, 2013.

SILVA, Tamara Oliveira et al. Avaliação da capacidade física e quedas em idosos ativos e sedentários da comunidade. Revista Brasileira de Clínica Médica, São Paulo, v. 8, n. 5, p. 392-398, set./out., 2010. 